\title{
Usefulness of computed tomography with air insufflation of the stomach prior to percutaneous endoscopic gastrostomy procedure
}

\author{
Kousaku Kawashima, ${ }^{1,2, *}$ Kyoichi Adachi, ${ }^{3}$ Koji Onishi, ${ }^{2}$ Kosuke Fukuda, ${ }^{2}$ Hideaki Kazumori, ${ }^{2}$ Yasuhiko Ohno, ${ }^{2}$ \\ Takao Katoh, ${ }^{1}$ Hiroki Sonoyama, ${ }^{1}$ Yasumasa Tada, ${ }^{1}$ Ryusaku Kusunoki, ${ }^{1}$ Akihiko Oka, ${ }^{1}$ Nobuhiko Fukuba, ${ }^{1}$ \\ Naoki Oshima, ${ }^{1}$ Takafumi Yuki, ${ }^{1}$ Shunji Ishihara' ${ }^{1}$ and Yoshikazu Kinoshita ${ }^{1}$ \\ 1Department of Internal Medicine II, Shimane University Faculty of Medicine, 89-1, Enya-cho, Izumo, Shimane 693-8501, Japan \\ 2Department of Internal Medicine, Matsue Seikyo General Hospital, Matsue, Shimane 690-8522, Japan \\ ${ }^{3}$ Health Center, Shimane Environment and Health Public Corporation, Matsue, Shimane 690-0012, Japan
}

We examined the results of computed tomography (CT) with and without air insufflation of the stomach prior to performing percutaneous endoscopic gastrostomy (PEG). We retrospectively analyzed 366 patients who underwent PEG. CT images obtained with and without air insufflation were examined for the presence or absence of contact between the gastric anterior wall and abdominal wall. PEG outcome based on CT findings was also examined. CT with and without air insufflation was performed in 272 and 94 patients, respectively. Contact between the gastric anterior wall and abdominal wall was shown in 254 (93.4\%) with and 45 $(47.9 \%)$ without air insufflation, all of whom underwent a successful PEG procedure. In patients without contact between the gastric anterior wall and abdominal wall, PEG was not successful in 3 of $49(6.1 \%)$ examined by CT without and 6 of $18(33.3 \%)$ examined with air insufflation $(p=0.004)$. Values for diagnostic accuracy for contact between the gastric anterior wall and abdominal wall shown by CT with and without air insufflation in successful PEG cases were 0.96 and 0.51 , respectively. In conclusion, CT with air insufflation more often revealed contact between the gastric anterior wall and abdominal wall as compared to CT without air insufflation, which may help to predict PEG procedure success.

Key Words: percutaneous endoscopic gastrostomy, computed tomography, air insufflation, complication

$\mathrm{P}$ ercutaneous endoscopic gastrostomy (PEG) procedures are widely performed for patients with swallowing dysfunction caused by a variety of disease conditions ${ }^{(1-3)}$ and found to be safe in about $95 \%$ of reported cases. ${ }^{(4,5)}$ However, several complications of a PEG procedure have been noted, such as bleeding, peritonitis, aspiration pneumonia, and iatrogenic penetration of other organs. ${ }^{(4-11)}$ Notably, false puncture of other organs is one of the most serious complications encountered during PEG. ${ }^{(9-11)}$ Abdominal computed tomography $(\mathrm{CT})$ performed prior to a PEG procedure has been recommended for assessment of the location of other organs surrounding the stomach and prevention of their false puncture..$^{(1,11-13)}$ Furthermore, CT applied after air expansion of the stomach is considered to be helpful to perform a successful gastrostomy procedure, as it provides a similar status of the stomach and other surrounding organs as that observed during a PEG procedure. However, there are few reports of the effectiveness of CT with an air-expanded stomach prior to PEG and there is no report of that compared to conventional CT without such air expansion. ${ }^{(14,15)}$ Moreover, the usefulness of $\mathrm{CT}$ with gastric expansion has not been examined in regard to assistance with a successful PEG procedure in comparison to CT without air insufflation. Therefore, we conducted this large-scale retrospective study to clarify the effectiveness of CT with air insufflation performed prior to a PEG procedure in comparison to conventional CT without air insufflation.

\section{Materials and Methods}

Study population. This study was approved by the ethical committee of Matsue Seikyo General Hospital. The study subjects were patients scheduled to undergo a PEG procedure at Matsue Seikyo General Hospital from April 2006 to September 2011. Written informed consent to perform PEG and related procedures was obtained from the patients and/or their family members. Those with a past history of gastrectomy or for whom a gastroscopy procedure could not be performed due to esophageal stenosis were excluded from the study. Thus, a total of 366 patients (151 men, 215 women; mean age 82.6 years) were analyzed. Age, sex, and underlying disease were also investigated as patient demographic factors.

CT with and without air insufflation of the stomach.

All study subjects were examined by abdominal CT with or without air insufflation of the stomach prior to the PEG procedure. That with air insufflation was performed immediately after inflation of the stomach with exactly $500 \mathrm{ml}$ of air injected via a gastric tube. Using CT images obtained with and without air insufflation, we investigated the presence or absence of contact between the gastric anterior wall (GAW) and abdominal wall (AW) in the epigastric area, as well as abnormal findings related to abdominal organs. Representative CT images showing contact between the GAW and AW obtained with and without air insufflation into the stomach are presented in Fig. 1. No potential contact between those could be observed when the transverse colon and/or liver were positioned in front of the stomach or with dislocation of the stomach to subcostal space.

Standard and special methods for PEG. PEG was routinely performed with a Kangaroo Seldinger PEG kit ${ }^{\mathrm{TM}}$ (Nippon Covidien Ltd., Tokyo, Japan). After confirmation of endoscopic transillumination and indentation of the stomach by external palpation, a 20-French PEG tube was inserted into the stomach using a modified introducer method, as previously reported. ${ }^{(16)}$

*To whom correspondence should be addressed. E-mail: kk461223@med.shimane-u.ac.jp 

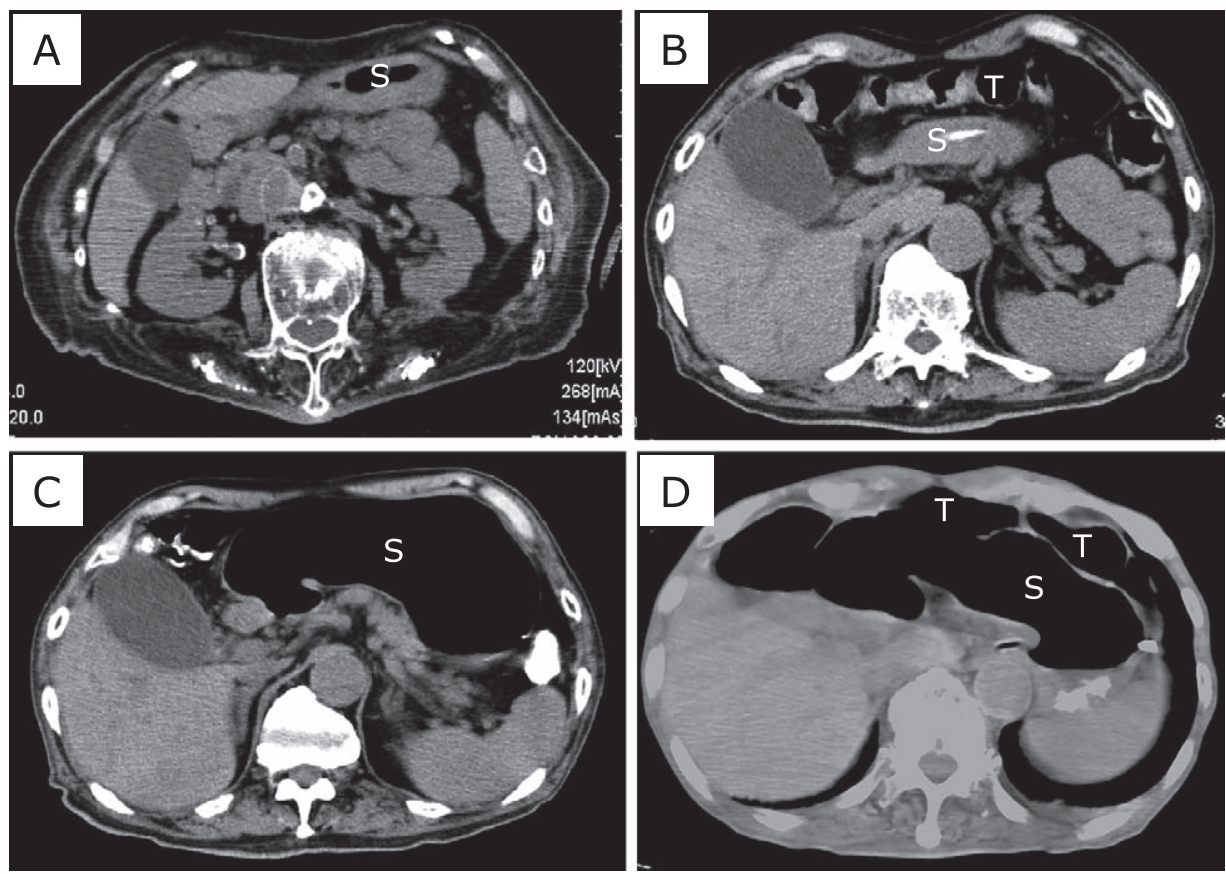

Fig. 1. Representative $C T$ images obtained with and without air insufflation of the stomach. (A) Contact between gastric anterior wall (GAW) and abdominal wall (AW), as shown by CT without air insufflation. (B) No contact between GAW and AW, as shown by CT without air insufflation. (C) Contact between GAW and AW, as shown by CT with air insufflation. (D) No contact between GAW and AW, as shown by CT with air insufflation. S, stomach; T, transverse colon.

When gastropexy was considered to be impossible, PEG was performed by a pull method using the Ponsky technique with a Safety PEG kit ${ }^{\mathrm{TM}}$ (Boston Scientific, Tokyo, Japan). ${ }^{(1,17,18)}$ When it was determined that a safety puncture using a standard PEG method as noted above would be impossible, we attempted special PEG methods that required additional resources, such as assistance from fluoroscopic imaging with contrast medium, colon preparation, or colonoscopy..$^{(14,19,20)}$

Statistical analysis. Initially, we determined the percentages of patients with the presence and absence of contact between the GAW and AW shown by CT with and without air insufflation. Based on the CT findings, we then examined the success of PEG with all methods. Utilizing these results, sensitivity, specificity, predictive values, and $\mathrm{CT}$ accuracy to show the presence of contact between the GAW and AW in relation to the success of PEG were determined for comparison of CT with and without air insufflation. Statistical analysis was performed using a chi-square test and Mann-Whitney's $U$ test, with $p<0.05$ considered to be statistically significant. All calculations were done with the SPSS statistical package (ver. 19.0, SPSS, Chicago, IL).

\section{Results}

Patients and CT findings. Of the 366 study subjects, 272 were examined by CT with air insufflation of the stomach and 94 underwent that without air insufflation prior to a PEG procedure. Characteristics and CT findings of patients who underwent CT with and without air insufflation are shown in Table 1 . The patient characteristics of age, sex, and underlying diseases were not significantly different between the groups. With regard to CT findings, the presence of contact between the GAW and AW was shown in $254(93.4 \%)$ patients with air insufflation and in 45 $(47.9 \%)$ without air insufflation, which were significantly different $(p<0.001)$.

Success of PEG according to CT findings. A flowchart presenting outcomes of the present PEG procedures according to the presence or absence of contact between the GAW and AW shown by $\mathrm{CT}$ are presented in Fig. 2 . In all patients with contact between the GAW and AW shown by CT with and without air insufflation, a standard PEG procedure was safely and successfully performed. As for the 18 patients without contact between the GAW and AW shown by CT with air insufflation, a standard PEG procedure was not attempted, because a related safe puncture was considered to be impossible. In $12(66.7 \%)$ of those cases, we were able to perform successful PEG only by use of special methods, while PEG was not successfully performed regardless of the method employed in the other $6(33.3 \%)$. On the other hand, in $40(81.6 \%)$ of 49 patients shown to not have contact between the GAW and AW by CT without air insufflation, standard PEG was successfully performed. In 6 of the remaining 9 patients, PEG was safely performed with the assistance of special methods.

Based on these results, we determined sensitivity, specificity, predictive values, and diagnostic accuracy for the presence of contact between the GAW and AW shown by CT with and without air insufflation of the stomach for a successful PEG procedure (Table 2). In cases of CT with air insufflation, sensitivity, negative predictive value (NPV), and accuracy were significantly superior as compared to those without air insufflation.

\section{Discussion}

Abdominal CT performed prior to a PEG procedure has been recommended for assessing the location of other organs surrounding the stomach and prevent their false puncture..$^{(1,11-13)}$ However, to the best of our knowledge, this is the first comparative study of the usefulness of CT with and without air insufflation prior to a PEG procedure.

Our results showed that $\mathrm{CT}$ with air insufflation prior to a PEG procedure more often revealed contact between the GAW and AW as compared to CT without air insufflation $(93.4 \%$ vs $47.9 \%)$. We found that $18(6.6 \%)$ of 272 of patients who underwent $C T$ with air insufflation had a high risk of false puncture of other organs 
Table 1. Characteristics and findings of patients who underwent CT with and without air insufflation of the stomach

\begin{tabular}{lccc}
\hline & CT with air insufflation & CT without air insufflation & $p$ value \\
\hline 1. Characteristics & 272 & 94 & \\
Number & $111 / 161$ & $40 / 54$ & 0.72 \\
Male/female & $82.5 \pm 10.0$ & $83.1 \pm 9.6$ & 0.72 \\
Mean age (years) & & & \\
Underlying disease & 68 & 20 & 0.47 \\
Cerebral infarction & 28 & 5 & 0.15 \\
Cerebral hemorrhage & 33 & 11 & 0.91 \\
Neurodegenerative disease & 37 & 10 & 0.46 \\
Dementia & 5 & 2 & 0.86 \\
Anoxic encephalopathy & 63 & 26 & 0.38 \\
Pneumonia & 13 & 5 & 0.83 \\
Heart disease & 25 & 15 & 0.11 \\
Others & & & $<0.001$ \\
2. CT findings & $254 / 18$ & $45 / 49$ & \\
Contact ${ }^{\dagger}$ (present/absent) &
\end{tabular}

${ }^{+}$Contact: presence of contact between gastric anterior wall and abdominal wall observed in CT images.
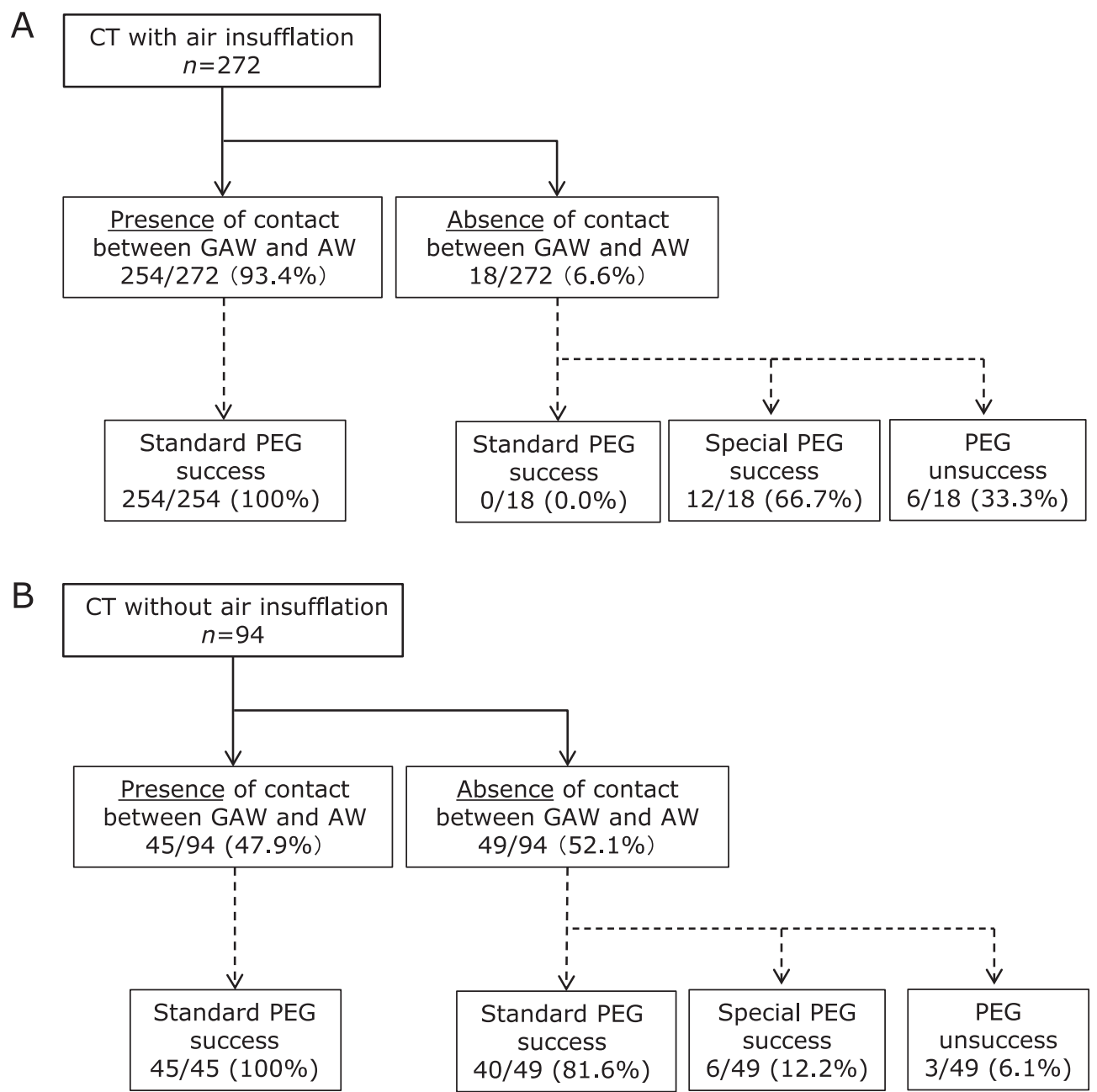

Fig. 2. Flowchart of outcomes of PEG procedures according to presence or absence of contact between gastric anterior wall and abdominal wall shown by CT. (A) CT with air insufflation. (B) CT without air insufflation. 
Table 2. Sensitivity, specificity, predictive values and accuracy of the presence of contact between the GAW and AW shown by CT for the success of PEG

\begin{tabular}{lccc}
\hline & CT with air insufflation & CT without air insufflation & $p$ value \\
\hline Sensitivity $(95 \% \mathrm{Cl})$ & $0.96(0.92-0.98)$ & $0.49(0.39-0.60)$ & $<0.001$ \\
Specificity $(95 \% \mathrm{Cl})$ & $1.00(0.54-1.00)$ & $1.00(0.29-1.00)$ & - \\
$\operatorname{PPV}^{\S}(95 \% \mathrm{Cl})$ & $1.00(0.99-1.00)$ & $1.00(0.92-1.00)$ & - \\
$\operatorname{NPV}^{\S}(95 \% \mathrm{Cl})$ & $0.33(0.13-0.59)$ & $0.06(0.01-0.17)$ & 0.004 \\
Accuracy $(95 \% \mathrm{Cl})$ & $0.96(0.92-0.98)$ & $0.51(0.41-0.62)$ & $<0.001$ \\
\hline
\end{tabular}

${ }^{\ddagger} \mathrm{Cl}$, Confidence interval. SPPV, positive predictive value; NPV, negative predictive value.

during PEG, because of absence of contact between the GAW and $\mathrm{AW}$, indicating that $\mathrm{CT}$ with air insufflation provides a more precise assessment of false puncture risk. In a previous report of CT with $300 \mathrm{ml}$ of air insufflation of the stomach, $15 \%$ of those patients had no contact shown between the GAW and AW, and were considered to be at high risk for undergoing a PEG procedure. ${ }^{(14)} \mathrm{We}$ consider that insufflation with $500 \mathrm{ml}$ of air is better for detecting high risk cases as compared to $300 \mathrm{ml}$.

Chang et al. ${ }^{(21,22)}$ proposed that an abdominal plain X-ray examination after $500 \mathrm{ml}$ of air insufflation into the stomach is useful to determine the abdominal puncture point used for PEG. Based on that proposal, we considered that CT with air insufflation at $500 \mathrm{ml}$ was useful to determine the abdominal puncture point and predict the success of PEG, as it provides a similar status of the stomach and other surrounding organs as observed during a PEG procedure. In the present study, sensitivity, NPV, and diagnostic accuracy of the presence of contact between the GAW and $\mathrm{AW}$ shown by $\mathrm{CT}$ with air insufflation was significantly superior for the success of PEG as compared to CT without air insufflation. In particular, the values for diagnostic accuracy for the presence of contact between the GAW and AW by CT with and without air insufflation were 0.96 and 0.51 , respectively. Thus, we concluded that CT with air insufflation is superior for prediction of the success of a PEG procedure as compared to that without air insufflation.

Furthermore, our results may suggest that CT with air insufflation is useful for deciding the appropriate PEG method for each case. If contact between the GAW and AW is confirmed by CT with as well as without air insufflation, we suggest that PEG with the standard method would be adequate, since that was safely and successfully performed in all such cases in our study. However, when no contact between the GAW and AW is confirmed by CT with air insufflation, we think that a standard PEG method should be avoided, as those patients are considered to have a high risk of false puncture of other organs during the PEG procedure. Rather, a special method is recommended and must be performed with close attention to avoid false puncture of other organs. In our study, $46(93.9 \%)$ of 49 patients without contact between the GAW and AW shown by CT without air insufflation underwent a successful PEG procedure. Of those successful cases, a standard PEG method that did not require any additional procedures was successfully performed in $40(81.6 \%)$. These results suggest that absence of contact between the GAW and AW shown by CT without air insufflation does not contribute to an appropriate choice of PEG method.

There are some limitations to this study. First, it was performed in a retrospective manner. Second, the patients were not randomly allocated to the CT with and without air insufflation groups. However, the patient characteristics were not significantly different between those groups. In addition, we did not investigate the adequate volume of air for insufflation of the stomach needed for success and safety, and an additional prospective study is necessary.

In conclusion, $\mathrm{CT}$ with air insufflation of the stomach prior to a PEG procedure can provide a more precise assessment of the risk of false puncture of other organs during the PEG procedure as compared to that without air insufflation. In addition, the findings obtained may contribute to prediction of success of PEG and data for making an appropriate choice in regard to PEG method.

\section{Abbreviations}

$\begin{array}{ll}\text { AW } & \text { abdominal wall } \\ \text { CT } & \text { computed tomography } \\ \text { GAW } & \text { gastric anterior wall } \\ \text { PEG } & \text { percutaneous endoscopic gastrostomy }\end{array}$

\section{Conflict of Interest}

No potential conflicts of interest were disclosed.

\section{References}

1 Gauderer MW. Percutaneous endoscopic gastrostomy-20 years later: a historical perspective. J Pediatr Surg 2001; 36: 217-219.

2 Iwamoto J, Honda A, Miyamoto Y, et al. Serum carnitine as an independent biomarker of malnutrition in patients with impaired oral intake. J Clin Biochem Nutr 2014; 55: 221-227.

3 Takaoka A, Sasaki M, Kurihara M, et al. Comparison of energy metabolism and nutritional status of hospitalized patients with Crohn's disease and those with ulcerative colitis. J Clin Biochem Nutr 2015; 56: 208-214.

4 Larson DE, Burton DD, Schroeder KW, DiMagno EP. Percutaneous endoscopic gastrostomy: indications, success, complications, and mortality in 314 consecutive patients. Gastroenterology 1987; 93: 48-52.

5 Mathus-Vliegen LM, Koning H. Percutaneous endoscopic gastrostomy and gastrojejunostomy: a critical reappraisal of patient selection, tube function and the feasibility of nutritional support during extended follow-up. Gastrointest Endosc 1999; 50: 746-754.

6 Kobayashi K, Cooper GS, Chak A, Sivak MV Jr, Wong RC. A prospective evaluation of outcome in patients referred for PEG placement. Gastrointest

Endosc 2002; 55: 500-506.

7 Figueiredo FA, da Costa MC, Pelosi AD, Martins RN, Machado L, Francioni E. Predicting outcomes and complications of percutaneous endoscopic gastrostomy. Endoscopy 2007; 39: 333-338.

8 Schrag SP, Sharma R, Jaik NP, et al. Complications related to percutaneous endoscopic gastrostomy (PEG) tubes. A comprehensive clinical review. $J$ Gastrointestin Liver Dis 2007; 16: 407-418.

9 Kinoshita Y, Udagawa H, Kajiyama Y, et al. Cologastric fistula and colonic perforation as a complication of percutaneous endoscopic gastrostomy. Surg Laparosc Endosc Percutan Tech 1999; 9: 220-222.

10 Lohiya GS, Tan-Figueroa L, Krishna V. Intermittent diarrhea as a delayed presentation of percutaneous endoscopic gastrostomy (PEG)-associated fistula. $J$ Am Board Fam Med 2010; 23: 681-684.

11 Friedmann R, Feldman H, Sonnenblick M. Misplacement of percutaneously inserted gastrostomy tube into the colon: report of 6 cases and review of the literature. J Parenter Enteral Nutr 2007; 31: 469-476.

12 Vogt W, Messmann H, Lock G, et al. CT-guided PEG in patients with un- 
successful endoscopic transillumination. Gastrointest Endosc 1996; 43: 138140.

13 Spelsberg FW, Hoffmann RT, Lang RA, et al. CT fluoroscopy guided percutaneous gastrostomy or jejunostomy without (CT-PG/PJ) or with simultaneous endoscopy (CT-PEG/PEJ) in otherwise untreatable patients. Surg Endosc 2013; 27: 1186-1195.

14 Sasaki T, Fukumori D, Sakai K, Sato M, Ohmori H, Yamamoto F. The safety and feasibility of percutaneous endoscopic gastrostomy placement. Hepatogastroenterology 2004; 51: 1062-1065.

15 Adachi Y, Akino K, Mita H, Kikuchi T, Endo T. Computed tomography just after endoscopy as the preoperative examination for safe percutaneous endoscopic gastrostomy. Digestion 2013; 88: 125-127.

16 Horiuchi A, Nakayama Y, Tanaka N, Fujii H, Kajiyama M. Prospective randomized trial comparing the direct method using a $24 \mathrm{Fr}$ bumper-buttontype device with the pull method for percutaneous endoscopic gastrostomy. Endoscopy 2008; 40: 722-726.
17 Ponsky JL, Gauderer MW. Percutaneous endoscopic gastrostomy: a nonoperative technique for feeding gastrostomy. Gastrointest Endosc 1981; 27: 9-11.

18 Ponsky JL, Gauderer MW. Percutaneous endoscopic gastrostomy: indications, limitations, techniques, and results. World J Surg 1989; 13: 165-170.

19 Silas AM, Pearce LF, Lestina LS, et al. Percutaneous radiologic gastrostomy versus percutaneous endoscopic gastrostomy: a comparison of indications, complications and outcomes in 370 patients. Eur J Radiol 2005; 56: 84-90.

20 Tominaga K, Saigusa Y, Ito S, Hirahata K, Nemoto Y, Maetani I. Percutaneous endoscopic gastrostomy with the aid of a colonoscope to avoid gastrocolic fistula formation. Endoscopy 2007; 39 Suppl 1: E112-E113.

21 Chang WK, Hsieh TY. Safety of percutaneous endoscopic gastrostomy in high-risk patients. J Gastroenterol Hepatol 2013; 28 Suppl 4: 118-122.

22 Chang WK, McClave SA, Yu CY, Huang HH, Chao YC. Positioning a safe gastric puncture point before percutaneous endoscopic gastrostomy. Int J Clin Pract 2007; 61: 1121-1125. 